\title{
Coronary artery calcium score and coronary computed tomography angiography for patients with asymptomatic polyvascular (non-coronary) atherosclerosis
}

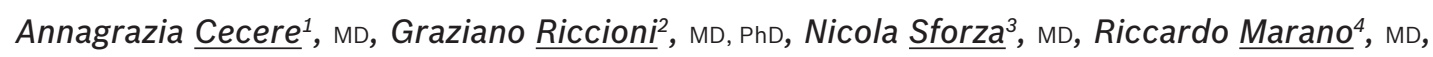
Giuseppe Guglielmi ${ }^{1,3}, \mathrm{MD}$

\begin{abstract}
INTRODUCTION The primary endpoint of this study was to determine the prevalence of coronary artery disease (CAD) and coronary artery calcium score (CACS) using coronary computed tomography angiography (CCTA) among patients with polyvascular atherosclerosis (PVA). Secondary endpoints were to evaluate the prevalence of cardiovascular risk factors and determine the predictors of PVA.

METHODS The presence of atherosclerotic disease was assessed using ultrasonographic vascular examination in 515 asymptomatic patients. All patients with presence of stenosis over $50 \%$ and moderate-to-severe cardiovascular risk profile underwent CCTA to identify atherosclerotic coronary disease.

RESULTS Among 515 participants, 143 patients had no evidence of atherosclerotic plaque. Of the 372 patients with atherosclerotic plaque, 184 patients had single-vessel disease, 111 patients had double-vessel disease and 77 patients had triple-vessel disease; among these patients, those who also presented with stenosis $>50 \%$ underwent CCTA. Coronary stenosis categories included: normal (6.4\%); haemodynamically insignificant (34.6\%); intermediate (28.7\%); significant (26.6\%); and total artery occlusion (3.7\%). Based on the coronary vessel involved, the patients were categorised as having single- $(41.0 \%)$, double- $(42.0 \%)$ or triple- $(17.0 \%)$ coronary disease. CACS was significantly higher in patients with double- or triple-vessel disease when compared to those with single-vessel disease.

CONCLUSION Our study confirmed not only the high sensitivity of CCTA for highlighting CAD, but also its negative predictive value for excluding the presence of coronary stenosis or ischaemia. We found good correlation between PVA and CACS, and were able to confirm the risk factors for PVA.
\end{abstract}

Keywords: coronary artery calcium score, coronary artery disease, coronary computed tomography angiography, polyvascular atherosclerosis

\section{INTRODUCTION}

Atherosclerosis, a systemic disease that affects the coronary, peripheral and cerebrovascular arteries, is the dominant cause of cardiovascular disease (CVD), including acute myocardial infarction (AMI), chronic heart failure, stroke and claudication, especially in Western countries. ${ }^{(1)}$ It primarily affects the intima in mediumsized and large arteries, resulting in intimal thickening, and may lead to luminal narrowing and inadequate blood supply. ${ }^{(2)}$ Mature atherosclerotic plaque typically consists of two main components, one of which is lipid-rich and soft, while the other is collagen-rich and hard. The flow-limiting potential of an intimal plaque may be modified by reactive changes in the underlying media and adventitia, which may attenuate (positive remodelling) or accentuate (negative remodelling) luminal obstruction and the consequent haemodynamic impact of the plaque. ${ }^{(3)}$

It is widely known that endothelial dysfunction is the first pathogenic event in the atherosclerotic process. ${ }^{(4)}$ Endothelial dysfunction, in fact, causes changes in vascular homoeostasis that make the vessel more vulnerable to dangerous events. ${ }^{(4)}$

On the basis of these considerations, cerebrovascular disease, peripheral arterial disease (PAD) and coronary artery disease (CAD) represent different faces of the same coin that have a common denominator in atherosclerosis. This consideration suggests a correlation between CAD and atherosclerotic involvement of other arterial districts, such as the carotid/aortic/ femoral district, called polyvascular atherosclerosis (PVA). The high prevalence of CVD in Western countries could be explained by the large spread of 'pro-atherogenic' lifestyles, such as cigarette smoking, a sedentary lifestyle and diets that are too rich in fats. ${ }^{(4)}$ Concomitant CAD and PAD is associated with a two- to threefold increased risk of CVD mortality. (1) Patients with PAD have more severe $C A D$, as manifested in a higher frequency of left main and multivessel $\mathrm{CAD},{ }^{(5)}$ physical function impairment and worse quality of life. ${ }^{(6)}$ These results were confirmed by Steg et al, ${ }^{(1)}$ who demonstrated that mortality and risk of future cardiac events increase progressively with extension of the atherosclerotic process to different parts of the body, highlighting that atherosclerosis is a systemic disease.

The Alliance study, ${ }^{(7)}$ Conducted on 9,783 patients who were hospitalised for AMI in France from 2000 to 2005, showed that PVA was an important predictor of prognosis. This result could be explained by not only the greater severity of the atherosclerotic process but also the prevalent diagnostic and treatment attitudes, which were more conservative for this subset of patients. Many studies have shown that patients with PVA are considered to be a risk category and, for this reason, are undertreated..$^{(8)}$

${ }^{1}$ Department of Radiology, University of Foggia, ${ }^{2}$ Intensive Cardiology Care Unit, San Camillo de Lellis Hospital, ${ }^{3}$ Department of Radiology, Scientific Institute Hospital, Casa Sollievo della Sofferenza, ${ }^{4}$ Department of Bioimaging and Radiological Sciences, Institute of Radiology, Catholic University, Italy

Correspondence: Prof Giuseppe Guglielmi, Department of Radiology, University of Foggia, Viale Luigi Pinto 1, 71100 Foggia, Italy. giuseppe.guglielmi@unifg.it 
Early diagnosis of PAD in patients with CAD should prompt aggressive risk factor modification to slow the progression of atherosclerosis, and prevent premature death, heart attack and stroke. The detection of CAD in patients with PVA is important to prevent cardiac mortality and morbidity. Recently, there has been an increased interest in atherosclerosis of the lower extremity arteries and its presence as multifocal disease (carotid/ aortic/femoral). However, awareness of non-CAD and its major prognostic implications in the general population and medical community remains relatively low. ${ }^{(9)}$

The primary endpoint of the present study was to determine the prevalence of coronary atherosclerotic lesions and coronary artery calcium score (CACS), detected using coronary computed tomography angiography (CCTA), in patients with PVA. Secondary endpoints were to evaluate the prevalence of cardiovascular risk factors in patients enrolled in the study and determine the predictors of PVA.

\section{METHODS}

This prospective study included 515 asymptomatic patients (age range 40-75 years) who underwent ultrasonographic vascular examination (UVE) at the Intensive Cardiology Care Unit of San Camillo de Lellis Hospital, Manfredonia, Foggia, Italy, between February 2013 and March 2014. The patients enrolled in the study were asymptomatic for CVD. The age range of 40-75 years was chosen as CVDs most often occur in patients in this age group.

During the study period, 730 potential asymptomatic participants underwent a routine UVE (carotid investigation of the extracranial carotid arteries [i.e. the common, internal and external carotid arteries], abdominal aorta and femoral district) in order to evaluate for atherosclerotic vascular involvement. Of these patients, 215 were not eligible for inclusion in the study. The most common reasons for ineligibility were: bilateral carotid artery occlusions $(\mathrm{n}=35)$; recent endovascular interventions $(n=77)$; monolateral endarterectomy $(n=63)$; presence of aortic abdominal aneurysm $(\mathrm{n}=28)$; and peripheral revascularisation $(\mathrm{n}=12)$.

Potential participants were considered to be asymptomatic for PVA (carotid arteries, abdominal aorta and femoral arteries) if they had never experienced a transient ischaemic attack, amaurosis fugax, stroke, abdominal pain or claudication. Patients were excluded from the study if they had symptomatic carotid, abdominal or peripheral artery diseases, current infectious or inflammatory disease, recent operations or endovascular interventions, bilateral carotid or femoral-popliteal occlusion, vascular stent implantation or monolateral/bilateral endarterectomy.

A patient was categorised as a smoker if he or she had smoked cigarettes, cigars or a pipe within the past 30 days. Body weight was measured using a balance scale. Body mass index was computed as the ratio of weight to the square of height $\left(\mathrm{kg} / \mathrm{m}^{2}\right)$.

Arterial hypertension was defined as blood pressure values $\geq 140 / 90 \mathrm{mmHg}$ measured at least twice and was assumed to be present in patients taking antihypertensive drugs. ${ }^{(10)}$ Diabetes mellitus was defined according to the 2013 clinical practice recommendations of the expert committee of the European Association for the Study of Diabetes and European Society of Cardiology (ESC). ${ }^{(11)}$ Hyperlipidaemia was defined as an elevation of low-density lipoprotein-cholesterol (LDL-C) levels above $130 \mathrm{mg} / \mathrm{dL}$ and was assumed to be present in all patients on lipid-lowering therapies. ${ }^{(12)}$

The diagnosis of PAD was classified in accordance with the American College of Cardiology Foundation/American Heart Association (ACCF/AHA) Task Force on Practice Guidelines. ${ }^{(13)}$ Stroke was defined as a neurological deficit after 24 hours, as evaluated by a neurologist or internist, according to the AHA American Stroke Association Stroke Council guidelines. ${ }^{(14)}$ History of myocardial infarction was defined according to the consensus document of the Joint ESC/American College of Cardiology Committee for the redefinition of myocardial infarction. ${ }^{(15)}$

The presence of atherosclerotic plaque, the percentage of stenosis and plaque type were assessed using UVE. ${ }^{(16)}$ PVA was defined as atherosclerotic plaque of the carotid arteries, abdominal aorta and femoral arteries. For this reason and on the basis of the affected territories, PVA was defined as: (a) carotid atherosclerosis (common, internal and external carotid); (b) abdominal aortic atherosclerosis; and (c) femoral atherosclerosis.

All patients with stenosis $>50 \%$ in one or more vascular districts and moderate-to-severe cardiovascular risk profile underwent CCTA to identify atherosclerotic coronary disease. Cardiovascular risk profile was calculated based on the ESC guidelines risk score carts (very high risk: > 10\%; high risk: $5 \%-10 \%$; moderate risk: $1 \%-5 \%$; low risk: $\leq 1 \%$ ) on the basis of the presence of documented CVD, diabetes mellitus and chronic kidney disease (CKD). ${ }^{(17)}$

The present study was conducted in collaboration with the Unit of Radiology at San Camillo de Lellis Hospital and the Department of Cardiac Surgery at Anthea Hospital, Bari, Italy. After being provided with information regarding the purpose of the study, all patients were requested to provide informed consent before participating in the study. The study was carried out in accordance with the Helsinki Declaration of 1975, as revised in 1983, and approved by the Ethics Review Committee and the Medical Direction of San Camillo de Lellis Hospital.

UVE was performed using a colour-coded ACUSON Sequoia ${ }^{\text {TM }}$ C512 carotid duplex ultrasound machine, with a 3.5-7.5-MHz linear and convex transducer (SIEMENS, New York, NY, USA). UVE included longitudinal and transverse examinations of the carotid arteries, abdominal aorta and femoralpopliteal arteries. Both diameter reductions were measured and calculated at the site of maximal stenosis. All measurements were made on frozen, enlarged images $(2 \times)$ at the end of a heart cycle (i.e. end diastole). ${ }^{(18)}$ UVE was performed by two cardiologists who specialised in vascular ultrasonography.

Atherosclerotic plaque was defined as focal echogenic structures encroaching into the vessel lumen, where the carotid intima media thickness (C-IMT) was $>1.2 \mathrm{~mm}$ (Fig. 1). C-IMT is the distance between the intima and the tunica media of the vessel examined. The high reproducibility of the examination, its low invasiveness and the close correlation in predicting adverse 


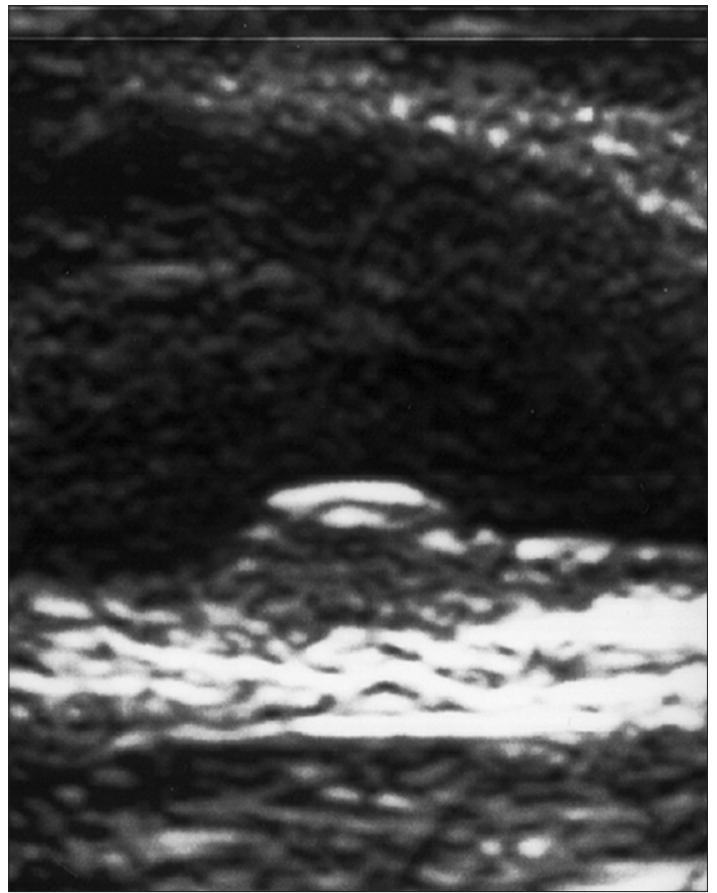

Fig. 1 Carotid ultrasonography image shows a carotid intima media thickness of $>1.2 \mathrm{~mm}$.

cardiac events make C-IMT an effective and reproducible marker of atherosclerosis. ${ }^{(19)}$ These cut-offs were chosen as they had been used in previous randomised clinical trials. ${ }^{(20)}$

After UVE, demographic and lifestyle characteristics, medical history, data from physical examination and laboratory examinations were collected (Box 1). Laboratory data was measured at the Analysis Laboratory of San Camillo de Lellis Hospital. All investigators and laboratory personnel were blinded to the patient's atherosclerotic disease.

The published literature has shown that the amount of calcium present in the coronary arteries is related to the degree of CAD and the consequent risk of developing adverse coronary events. ${ }^{(21)}$ It is also widely known that coronary calcifications share the same risk factors as atherosclerosis, and that changes in lifestyle and the introduction of lipid-lowering drugs may reduce their levels. ${ }^{(22)}$

Proceeding from this premise, Agatston et al assessed the amount of calcium in the coronary arteries using CCTA. In the past few years, this noninvasive measurement has been confirmed as a promising technique for identifying clinical and subclinical coronary stenoses. ${ }^{(23)}$ In particular, the risk of developing cardiovascular events has been shown to increase significantly when the Agatston score exceeds a value of 400. ${ }^{(24)}$

In our study, in accordance with standardised protocols, CACS was performed prior to coronary angiography. In fact, calcified atherosclerotic plaques can be displayed on CCTA even without the use of endovenous contrast agents. Investigators who were blinded to the patient characteristics conducted offline analysis using automated computerised software programs that employed the Agatston scoring method, ${ }^{(23)}$ with a threshold of $130 \mathrm{HU} .{ }^{(25)}$ The calcium score percentile based on age and gender was calculated using CACS distributions from the Multi-Ethnic Study of Atherosclerosis. ${ }^{(26)}$ This was performed using a web-based
Box 1. Patient demographic characteristics and clinical/ laboratory information collected.

- Demographic and lifestyle

Age, gender, height, weight, cigarette-smoking

\section{- Medical history}

Hypertension, diabetes mellitus, family history of

atherosclerosis, dyslipidaemia, prior acute myocardial infarction, angina pectoris, peripheral arterial disease, prior cerebral accident, current medications

\section{- Physical examination}

Blood pressure, body mass index

\section{- Laboratory investigations}

Total cholesterol, high-density lipoprotein-cholesterol, lowdensity lipoprotein-cholesterol, triglycerides, high-sensitivity C-reactive protein, fibrinogen, uric acid

calculator (http://www.mesa-nhlbi.org/Calcium/input.aspx). For patients $<45$ years old, ' 45 years' was used for the calculation of the calcium score percentile.

CCTA was conducted during contrast enhancement using prespecified protocols, as recommended by the scanner's manufacturers, during a single breath hold with prospective electrocardiographic gating, as appropriate. ${ }^{(27)}$ According to standardised protocols, CCTA is performed during inspiratory apnoea and an elevated heart rate can limit the spatial resolution of its image. ${ }^{(24)}$ For this reason, for all patients evaluated using CCTA, examination was done with heart rate in the range of 60-65 beats per minute. All CCTA examinations were assessed by two observers with extensive training in cardiac imaging. The examiners - radiologists and cardiologists, in particular - were blinded to the patient's characteristics. The presence of two specialists for the execution and reporting of the examination enabled, on one hand, integration of medical knowledge and, on the other, greater emphasis on scientific interest vis-à-vis CCTA. Angiograms were reported using the 15-segment model. ${ }^{(28)}$

We defined significant stenosis due to CAD as stenosis $>70 \%$ in one or more major epicardial vessels, or stenosis $>50 \%$ in the left main stem. Luminal cross-sectional area stenoses were classified as normal ( $<10 \%$ stenosis), haemodynamically insignificant $(10 \%-49 \%)$, intermediate $(50 \%-70 \%)$, significant $(\geq 70 \%)$, or total or subtotal occlusion $(100 \%) .{ }^{(29)}$ Study participants were grouped into three atherosclerotic categories on the basis of the affected territories: carotid, aortic and femoral.

The statistical significance of differences was examined using analysis of variance for continuous variables and chi-square test for categorical variables. Associations among demographic variables, lifestyle, medical history and laboratory characteristics, as well as the three atherosclerotic categories were examined using logistic regression models. A p-value $<0.05$ was considered to be statistically significant. Data was analysed using SPSS version 10.0 for Windows (SPSS Inc, Chicago, IL, USA).

\section{RESULTS}

The mean or percentage values for the various demographic variables and established CVD risk factors are summarised by 
Table I. Demographic characteristics and cardiovascular risk factors of patients with polyvascular disease $(\mathbf{n}=\mathbf{3 7 2}$ ).

\begin{tabular}{|c|c|c|c|c|c|c|c|}
\hline \multirow[t]{2}{*}{ Variable } & \multicolumn{3}{|c|}{ Single-vessel disease } & \multicolumn{3}{|c|}{ Double-vessel disease } & \multirow{2}{*}{$\begin{array}{c}\begin{array}{c}\text { Triple-vessel } \\
\text { disease }\end{array} \\
\text { Carotid aortic } \\
\text { femoral }(n=77)\end{array}$} \\
\hline & $\begin{array}{l}\text { Carotid } \\
(n=98)\end{array}$ & $\begin{array}{c}\text { Aortic } \\
(n=32)\end{array}$ & $\begin{array}{l}\text { Femoral } \\
(n=54)\end{array}$ & $\begin{array}{l}\text { Carotid } \\
\text { aortic } \\
(n=48)\end{array}$ & $\begin{array}{l}\text { Carotid } \\
\text { femoral } \\
(n=40)\end{array}$ & $\begin{array}{c}\text { Aortic } \\
\text { femoral } \\
(n=23)\end{array}$ & \\
\hline \multicolumn{8}{|l|}{ Demographic } \\
\hline Age $(y r)^{*}$ & $62.6 \pm 10.2$ & $61.1 \pm 11.7$ & $60.4 \pm 12.7$ & $62.5 \pm 13.2$ & $60.6 \pm 12.3$ & $61.0 \pm 11.6$ & $65.3 \pm 10.3$ \\
\hline Male & 68.2 & 45.0 & 81.3 & 65.2 & 59.3 & 76.4 & 78.0 \\
\hline $\operatorname{BMI}\left(\mathrm{kg} / \mathrm{m}^{2}\right)^{*}$ & $26.0 \pm 3.2$ & $28.0 \pm 2.1$ & $28.6 \pm 2.1$ & $28.6 \pm 2.1$ & $26.0 \pm 3.2$ & $28.0 \pm 2.1$ & $28.6 \pm 2.1$ \\
\hline Smoking & 21.0 & 25.0 & 24.0 & 34.0 & 41.0 & 32.0 & 53.0 \\
\hline \multicolumn{8}{|l|}{ Medical history } \\
\hline Hypertension & 38.0 & 41.0 & 40.0 & 55.0 & 48.0 & 51.0 & 67.0 \\
\hline Diabetes mellitus & 15.0 & 10.0 & 11.0 & 28.0 & 24.0 & 29.0 & 44.0 \\
\hline Hyperlipidaemia & 48.0 & 45.0 & 35.0 & 58.0 & 55.0 & 59.0 & 83.0 \\
\hline \multicolumn{8}{|l|}{ Family history } \\
\hline Atherosclerosis & 12.0 & 7.0 & 18.0 & 22.0 & 12.0 & 17.0 & 33.0 \\
\hline Stroke & 10.0 & 5.0 & 9.0 & 11.0 & 5.0 & 9.0 & 22.0 \\
\hline CKD & 1.0 & 1.0 & 2.0 & 4.0 & 1.0 & 3.0 & 11.0 \\
\hline
\end{tabular}

Data presented as percentages unless otherwise stated. *Data presented as mean \pm standard deviation. AMI: acute myocardial infarction; BMI: body mass index; CKD: chronic kidney disease

Table II. Inflammatory marker and plasma lipid levels of patients with polyvascular disease $(n=372)$.

\begin{tabular}{|c|c|c|c|c|c|c|c|}
\hline \multirow[t]{2}{*}{ Variable } & \multicolumn{3}{|c|}{$\begin{array}{l}\text { Single vessel disease } \\
\qquad(n=184)\end{array}$} & \multicolumn{3}{|c|}{$\begin{array}{l}\text { Double vessel disease } \\
\qquad(n=111)\end{array}$} & \multirow{2}{*}{$\begin{array}{c}\text { Triple vessel } \\
\text { disease }(n=77)\end{array}$} \\
\hline & Carotid & Aortic & Femoral & $\begin{array}{l}\text { Carotid } \\
\text { aortic }\end{array}$ & $\begin{array}{l}\text { Carotid } \\
\text { femoral }\end{array}$ & $\begin{array}{l}\text { Aortic } \\
\text { femoral }\end{array}$ & \\
\hline TOT-C (mg/dL) & $171.1 \pm 12.8$ & $168.3 \pm 1.8$ & $170.6 \pm 10.7$ & $230.1 \pm 32.1$ & $228.5 \pm 32.1$ & $225.0 \pm 30.2$ & $211.2 \pm 15.7$ \\
\hline HDL-C (mg/dL) & $47.1 \pm 4.3$ & $46.5 \pm 5.6$ & $46.4 \pm 4.3$ & $44.1 \pm 7.5$ & $45.2 \pm 5.4$ & $43.1 \pm 7.2$ & $48.1 \pm 5.5$ \\
\hline LDL-C (mg/dL) & $120.1 \pm 8.4$ & $119.9 \pm 7.3$ & $121.3 \pm 7.5$ & $163.5 \pm 38.2$ & $160.2 \pm 40.1$ & $161.3 \pm 40.9$ & $165.2 \pm 30.1$ \\
\hline TG (mg/dL) & $122.1 \pm 20.7$ & $121.2 \pm 18.8$ & $120.6 \pm 19.5$ & $166.2 \pm 39.2$ & $160.1 \pm 40.2$ & $161.2 \pm 39.1$ & $180.2 \pm 32.5$ \\
\hline Uric acid $(\mu \mathrm{mol} / \mathrm{L})$ & $238.8 \pm 71.3$ & $242.6 \pm 68.3$ & $229.4 \pm 69.3$ & $317.5 \pm 85.5$ & $312.5 \pm 86.5$ & $307.5 \pm 91.6$ & $355.1 \pm 98.5$ \\
\hline hs-CRP (g/dL) & $2.5 \pm 1.2$ & $2.3 \pm 1.2$ & $2.4 \pm 1.1$ & $3.8 \pm 1.7$ & $3.6 \pm 1.8$ & $3.6 \pm 1.8$ & $4.8 \pm 2.1$ \\
\hline Fibrinogen (mg/dL) & $285.4 \pm 93.7$ & $278.1 \pm 90.2$ & $278.4 \pm 89.6$ & $349.5 \pm 80.6$ & $347.1 \pm 77.1$ & $367.3 \pm 79.2$ & $412.6 \pm 85.4$ \\
\hline $\begin{array}{l}\text { Coronary artery } \\
\text { calcium score }\end{array}$ & $142 \pm 122$ & $145 \pm 126$ & $139 \pm 115$ & $467 \pm 215$ & $422 \pm 234$ & $444 \pm 216$ & $667 \pm 195$ \\
\hline
\end{tabular}

All data presented as mean \pm standard deviation. HDL-C: high-density lipoprotein cholesterol; hs-CRP: high-sensitivity C-reactive protein; LDL-C: low-density lipoprotein cholesterol; TG: triglycerides; TOT-C: total cholesterol

category of PVA in Table I. Among the 515 patients enrolled in the study, 372 patients had atherosclerotic plaque, while 143 patients only had increased C-IMT ( $<1.2 \mathrm{~mm})$. As indicated earlier, only patients with ultrasonographic evidence of stenosis $>50 \%$ were subjected to further evaluations (e.g. CACS and CCTA) and, therefore, the 143 patients with C-IMT $<1.2 \mathrm{~mm}$ were excluded from subsequent imaging examinations.

Drug use for the treatment of several cardiovascular risk factors was comparable among the patient groups. The enrolled patients were also homogeneous for the presence of risk factors and extent of medical treatment.

Among the 372 patients with atherosclerotic plaque, 184 had single-vessel disease (i.e. atherosclerotic plaque localised only in the carotid, aortic or femoral district). The remaining 188 patients, who had either double- $(\mathrm{n}=111)$ or triple-vessel $(n=77)$ disease with ultrasonographic stenosis $>50 \%$, underwent
CCTA. Categories of coronary stenosis in this subset of patients included: normal $(n=12,6.4 \%)$; haemodynamically insignificant (i.e. non-obstructive) $(\mathrm{n}=65,34.6 \%)$; intermediate $(\mathrm{n}=54$, $28.7 \%)$; significant ( $\mathrm{n}=50,26.6 \%)$; and total artery occlusion $(\mathrm{n}=7,3.7 \%$ ). Based on the coronary vessels involved in this subset, $77(41.0 \%)$ patients had single coronary disease (involving the left coronary artery, right coronary artery and proximal circumflex artery), 79 (42.0\%) had double coronary disease and $32(17.0 \%)$ had triple coronary disease.

The odds ratio and 95\% confidence interval (Table II) of selected risk factors for PVA, including inflammatory markers and plasma lipid levels, indicate the extent of polyvascular disease in the study population. CACS was significantly higher among patients with double- $(p<0.01)$ or triple-vessel disease $(p<0.001)$, as compared to those with single-vessel disease. In multivariate-adjusted models, patients with elevated levels of 
inflammatory factors, plasma lipids and CACS were significantly more likely to have PVA ( $p<0.01$ for high-sensitivity C-reactive protein [hs-CRP], uric acid, fibrinogen, total cholesterol, LDL-C, triglycerides; $p<0.001$ for CACS; Table II).

The prevalence of cardiovascular risk factors was significantly higher among patients with PVA in three atherosclerotic districts as compared to those with PVA in one or two districts $(p<0.001)$. In particular, hypertension $(\mathrm{p}<0.001)$, hyperlipidaemia $(p<0.001)$, angina or AMI $(p<0.001)$ and CKD $(p<0.001)$ were the most important risk factors for PVA. The prevalence of diabetes mellitus ( $p<0.01)$, cigarette smoking $(p<0.01)$, and a family history of atherosclerosis $(p<0.01)$ and stroke $(p<0.01)$ were also statistically significant. The prevalence of cardiovascular risk factors in patients with PVA in two districts versus one district was statistically significant for variables such as cigarette smoking, hypertension, diabetes mellitus, hyperlipidaemia and CKD ( $p<0.01$ ); conversely, factors such as a family history of angina, myocardial infarction, atherosclerosis and stroke were not statistically significant $(p=0.62)$.

Inflammatory markers (e.g. uric acid, hs-CRP and fibrinogen) were all significantly higher in patients with PVA in three districts vs. one or two districts $(p<0.001)$. In addition, plasma lipids (total cholesterol, LDL-C and triglycerides) were significantly higher among patients with two or three PVA lesions as compared to those with atherosclerosis at only one site $(p<0.01)$.

\section{DISCUSSION}

Atherosclerosis is the leading cause of morbidity and mortality worldwide, and despite ongoing efforts to extend primary and secondary prevention for high-risk individuals, it is expected to be the primary cause of death until the year 2020. ${ }^{(30)}$ Atherothrombotic diseases are currently referred to as polyvascular disease. ${ }^{(31)}$

Increased awareness of the spread of atherosclerosis-related diseases has led to advances in the development of techniques that enable early diagnosis and appropriate cardiovascular risk stratification. The study of atherosclerotic plaques can be performed using invasive methods, such as intravascular ultrasonography, and intracoronary optical coherence tomography, as well as noninvasive methods such as ultrasonography, magnetic resonance imaging and CCTA. Based on the assumption that atherosclerosis is a polyvascular pathology by definition, the use of all these methods has allowed, over the years, correlation of the presence of atherosclerotic lesions in different parts of the body and the identification of effective subclinical markers.

The present study, which was part of this line of research, confirms the role of CCTA in providing high-resolution images of the coronary anatomy. ${ }^{(32)}$ The results of our study were within expectations, as we found that a high prevalence of CAD was detected by CCTA in patients with PVA involving two or three atherosclerotic districts, along with coronary stenosis $>50 \%$ and high or very high cardiovascular risk scores. This data emphasises the diagnostic accuracy of the technique, confirming, on one hand, the high sensitivity of CCTA in highlighting CAD and, on the other, the correlation between the two noninvasive approaches of ultrasonography and CCTA.
Our result is in perfect agreement with the published literature. In fact, a study conducted by Cohen et al ${ }^{(33)}$ compared the presence of carotid disease, evaluated using ultrasonography, and the presence of CAD, evaluated using CCTA. It showed that carotid plaque, in conjunction with C-IMT, correlates closely with the severity of coronary calcifications identified using CCTA.

We also demonstrated a good correlation between CACS and PVA. CACS correlates significantly with the degree of disease and the number of vessels involved, and was associated with extension and severity of CAD. Therefore, our study also emphasised the high positive predictive value of PVA, as evaluated by ultrasonography, for predicting the presence of coronary atherosclerosis. Indeed, higher values of CACS were demonstrated in patients with double- and triple-vessel disease in our study.

Furthermore, our study confirmed the important role of CACS, when evaluated using CCTA, as an indicator that determines the risk of cardiac events. This result is in perfect agreement with data in the literature, which demonstrates that the absence of coronary calcification is related to a better prognosis and lower risk of developing coronary events. This parameter is easy to perform, inexpensive and easily reproducible. ${ }^{(34)}$ Indeed, CACS has received a recommendation of Ila by ACCF/AHA for risk stratification in asymptomatic individuals in the intermediate risk class (Framingham risk score 10\%-20\%). ${ }^{(35)}$ In patients at low or high cardiovascular risk, evaluation of CACS is not required.

However, CACS is a parameter that continues to have many uncertainties associated with it. First, a high value of CACS is not closely related to equally significant coronary stenosis. Second, the absence of a high value of CACS does not completely rule out the possibility of CAD and, conversely, CACS cannot assess those plaques that are non-calcified or partially calcified. ${ }^{(36)}$ For this reason, a major limitation regarding the diagnostic accuracy of CCTA is coronary calcifications. The presence of calcium in the coronary arteries (in particular, CACS $\geq 400$ or CACS $\geq 600$ ) significantly reduces the specificity of CCTA. ${ }^{(36)}$ On the basis of this finding and the results obtained from our study, in which patients with double- and triple-vessel disease obtained a higher CACS, we confirmed that CCTA has a negative predictive value, as widely described in the scientific literature. ${ }^{(37)}$ It has been widely demonstrated that the sensitivity of CCTA in identifying severe stenosis is very high; on the contrary, the specificity tends to be lower due to the tendency of CCTA to overestimate stenosis, especially in calcified plaques. ${ }^{(24)}$ CCTA is, in fact, indicated for patients with low-to-intermediate risk of CAD because a negative result could exclude, with significant probability, the presence of CAD. Equally, in patients at high risk for CAD, CCTA is unnecessary not only because it cannot add any information to the high pretest probability of $\mathrm{CAD}$, but also as it may require a subsequent coronary angiography, which is still considered to be the gold standard for the identification of coronary stenosis.

However, in recent years, renewed interest in the development of new technologies has expanded the clinical application of CCTA. The combined use of CCTA as an imaging technique and for the measurement of fractional flow reserve (FFR) provides 
more information in patients with intermediate stenosis. ${ }^{(38)}$ The FFR is defined as the ratio between maximal flow in the stenotic vessel and maximal flow threshold in a normal vessel. A value of FFR $<0.8$ is considered to be suggestive of ischaemia. ${ }^{(39)}$ For this reason, patients with intermediate coronary stenosis and $\mathrm{FFR}<0.8$ can benefit from revascularisation; conversely, the presence of FFR $>0.8$ allows the adoption of conservative medical therapy.

Noninvasive imaging methods have been used to evaluate early signs of atherosclerosis. However, brightness-mode ultrasonography and cardiac magnetic resonance imaging do not enable complete evaluation of the coronary arteries. ${ }^{(40,41)}$ Conventional coronary angiography and myocardial perfusion imaging imperfectly depict atherosclerosis, whether in its earlier stages, or when the disease is mature but has not yet compromised luminal integrity by means of positive remodelling.

Our study confirms the important role of plasma lipid profiles and inflammatory markers in the pathogenesis of the atherosclerotic process. A significantly higher serum concentration of total cholesterol, LDL-C, triglycerides and uric acid was seen among patients with PVA. For this reason, the administration of lipid-lowering therapy would reduce the progression of carotid atherosclerotic plaque. These findings suggest that the first and earliest manifestation of the atherosclerotic process, characterised by plaque, depends partly on the presence of an abnormal plasma lipid profile, with high concentrations of LDL-C and triglycerides. The plasma lipids pattern of patients with PVA was associated with an abnormal lipid profile that had significantly higher concentrations of LDL-C and triglycerides. We also identified a strong positive association between values of the inflammatory markers tested in our study and PVA, a finding that is in agreement with other studies in the literature. ${ }^{(42)}$ In particular, a progressive increase was found to be related to the districts of interest of atherosclerotic plaque. This increase was independent of variables such as age, body mass index and plasma lipid levels on logistic regression analyses. This strong positive relationship may represent an important indicator of the oxidative stress and proinflammatory milieu involved in the atherosclerotic process.

Similar to earlier studies, we confirmed that arterial hypertension, diabetes mellitus, cigarette smoking, hyperlipidaemia, angina and myocardial infraction were the strongest and most significant risk factors for the development of PVA. Hypertension and diabetes mellitus, in particular, have been shown to contribute to the remodelling of the arterial wall. These results were in accord with the findings of a community-based study in Taiwan involving 3,602 participants, which demonstrated that hypertension was a major determinant of carotid atherosclerosis, particularly among patients aged over 35 years. $^{(43)}$

In summary, the present study confirmed the known risk factors of PVA, such as hypertension, diabetes mellitus, cigarette smoking, a positive family history of atherosclerosis or stroke and elevated inflammatory markers (e.g. uric acid, hs-CRP and fibrinogen), which may be used to create the risk factor profile of individuals at the highest risk of developing CAD, as evaluated with CCTA. However, also noteworthy was the negative predictive value of CCTA for excluding the presence of coronary stenosis or ischaemia. ${ }^{(39)}$ These findings suggest that the use of CCTA may be erroneous and off-label for asymptomatic patients with PVA. Notwithstanding such reservations, in view of the spread of CVD and the importance of early detection to undertake targeted therapy, the clinical application of CCTA among patients with PVA is likely to rise. Further trials are warranted to extend the use of CCTA to other subsets of patients who are at risk of CAD.

\section{REFERENCES}

1. Steg PG, Bhatt DL, Wilson PW, et al; REACH Registry Investigators. One-year cardiovascular event rates in outpatients with atherothrombosis. JAMA 2007; 297:1197-206

2. Libby P. Inflammation in atherosclerosis. Nature $2002 ; 420: 868-74$.

3. Vink A, Schoneveld AH, Richard W, et al. Plaque burden, arterial remodeling and plaque vulnerability: determined by systemic factors? J Am Coll Cardiol 2001; 38:718-23.

4. Ciccone MM, Bilianou E, Balbarini A, et al. Task force on: 'Early markers of atherosclerosis: influence of age and sex'. J Cardiovasc Med (Hagerstown) 2013; 14:757-66.

5. Moussa ID, Jaff MR, Mehran R, et al. Prevalence and prediction of previously unrecognized peripheral arterial disease in patients with coronary artery disease: the Peripheral Arterial Disease in Interventional Patients Study. Catheter Cardiovasc Interv 2009; 73:719-24.

6. Wilson WR, Fitridge RA, Weekes AJ, et al. Quality of life of patients with peripheral arterial disease and chronic stable angina. Angiology 2012; 63:223-8.

7. Meizels A, Zeitoun DM, Bataille V, et al; ALLIANCE Investigators on behalf of the working group on Epidemiology of the French Society of Cardiology. Impact of polyvascular disease on baseline characteristics, management and mortality in acute myocardial infarction. The Alliance project. Arch Cardiovasc Dis 2010; 103:207-14.

8. McDermott MM, Mehta S, Ahn H, Greenland P. Atherosclerotic risk factors are less intensively treated in patients with peripheral arterial disease than in patients with coronary artery disease. J Gen Intern Med 1997; 12:209-15.

9. Gallino A, Aboyans V, Diehm C, et al; European Society of Cardiology Working Group on Peripheral Circulation. Non-coronary atherosclerosis. Eur Heart J 2014; 35:1112-9.

10. Mancia G, Fagard R, Narkiewicz K, et al. 2013 ESH/ESC guidelines for the management of arterial hypertension: the Task Force for the Management of Arterial Hypertension of the European Society of Hypertension (ESH) and of the European Society of Cardiology (ESC). Eur Heart J 2013; 34:2159-219.

11. Authors/Task Force Members, Rydén L, Grant PJ, et al. ESC Guidelines on diabetes, pre-diabetes, and cardiovascular diseases developed in collaboration with the EASD: the Task Force on diabetes, pre-diabetes, and cardiovascular diseases of the European Society of Cardiology (ESC) and developed in collaboration with the European Association for the Study of Diabetes (EASD). Eur Heart J 2013; 34:3035-87.

12. National Cholesterol Education Program (NCEP) Expert Panel on Detection, Evaluation, and Treatment of High Blood Cholesterol in Adults (Adult Treatment Panel III). Third Report of the National Cholesterol Education Program (NCEP) Expert Panel on Detection, Evaluation, and Treatment of High Blood Cholesterol in Adults (Adult Treatment Panel III) final report. Circulation 2002; 106:3143-421.

13. Rooke TW, Hirsch AT, Misra S, et al; Society for Cardiovascular Angiography and Interventions; Society of Interventional Radiology; Society for Vascular Medicine; Society for Vascular Surgery. 2011 ACCF/AHA Focused Update of the Guideline for the Management of Patients with Peripheral Artery Disease (updating the 2005 guideline): a report of the American College of Cardiology Foundation/American Heart Association Task Force on Practice Guidelines. J Am Coll Cardiol 2011; 58:2020-45.

14. Goldstein LB, Adams R, Alberts MJ, et al; American Heart Association/American Stroke Association Stroke Council; Atherosclerotic Peripheral Vascular Disease Interdisciplinary Working Group; Cardiovascular Nursing Council; Clinical Cardiology Council; Nutrition, Physical Activity, and Metabolism Council; Quality of Care and Outcomes Research Interdisciplinary Working Group; American Academy of Neurology. Primary prevention of ischemic stroke: a guideline from the American Heart Association/American Stroke Association Stroke Council: cosponsored by the Atherosclerotic Peripheral Vascular Disease Interdisciplinary Working Group; Cardiovascular Nursing Council; Clinical Cardiology Council; Nutrition, Physical Activity, and Metabolism Council; and the Quality of Care and Outcomes Research Interdisciplinary Working Group: the American Academy of Neurology affirms the value of this guideline. Stroke 2006; 37:1583-633. 
15. Thygesen K, Alpert JS, Jaffe AS, et al; Writing Group on behalf of the Joint ESC/ACCF/AHA/WHF Task Force for the Universal Definition of Myocardia Infarction. Third universal definition of myocardial infarction. Glob Heart 2012 7:275-95.

16. Stary HC, Chandler AB, Dinsmore RE, et al. A definition of advanced types of atherosclerotic lesions and a histological classification of atherosclerosis. A report from the Committee on Vascular Lesions of the Council on Arteriosclerosis, American Heart Association. Arterioscler Thromb Vasc Biol $1995 ; 15: 1512-31$

17. Perk J, De Backer G, Gohlke H, et al; European Association for Cardiovascula Prevention \& Rehabilitation (EACPR); ESC Committee for Practice Guidelines (CPG). European Guidelines on cardiovascular disease prevention in clinical practice (version 2012). The Fifth Joint Task Force of the European Society o Cardiology and Other Societies on Cardiovascular Disease Prevention in Clinical Practice (constituted by representatives of nine societies and by invited experts). Eur Heart J 2012; 33:1635-701.

18. Kanters SD, Algra A, van Leeuwen MS, Banga JD. Reproducibility of in vivo carotid intima-media thickness measurements: a review. Stroke 1997; 28:665-71.

19. Wikstrand J. Methodological considerations of ultrasound measurement of carotid artery intima-media thickness and lumen diameter. Clin Physiol Func Imaging 2007; 27:341-45.

20. Crouse JR 3rd, Byington RP, Bond MG, et al. Pravastatin, lipids and atherosclerosis in the carotid arteries (PLAC-II). Am J Cardiol 1995; 75:455-9.

21. Margolis JR, Chen JT, Kong Y, et al. The diagnostic and prognostic significance of coronary artery calcification. A report of 800 cases. Radiology 1980 137:609-16

22. Greenland $\mathrm{P}$, Bonow RO, Brundage $\mathrm{BH}$, et al; American College of Cardiology Foundation Clinical Expert Consensus Task Force (ACCF/AHA Writing Committee to Update the 2000 Expert Consensus Document on Electron Beam Computed Tomography); Society of Atherosclerosis Imaging and Prevention Society of Cardiovascular Computed Tomography. ACCF/AHA 2007 clinica expert consensus document on coronary artery calcium scoring by computed tomography in global cardiovascular risk assessment and in evaluation of patients with chest pain: a report of the American College of Cardiology Foundation Clinical Expert Consensus Task Force (ACCF/AHA Writing Committee to Update the 2000 Expert Consensus Document on Electron Beam Computed Tomography) developed in collaboration with the Society of Atherosclerosis Imaging and Prevention and the Society of Cardiovascular Computed Tomography. J Am Coll Cardiol 2007; 49:378-402.

23. Agatston AS, Janowitz WR, Hildner FJ, et al. Quantification of coronary artery calcium using ultrafast computed tomography. J Am Coll Cardiol 1990; 15:827-32.

24. Eckert J, Schmidt M, Magedanz A, Voigtländer T, Schmermund A. Coronary CT angiography in managing atherosclerosis. Int J Mol Sci 2015; 16:3740-56.

25. Nicholls SJ, Tuzcu EM, Wolski K, et al. Coronary artery calcification and changes in atheroma burden in response to established medical therapies. J Am Coll Cardiol 2007; 49:263-70.

26. McClelland RL, Chung H, Detrano R, Post W, Kronmal RA. Distribution of coronary artery calcium by race, gender, and age: results from the Multi-Ethnic Study of Atherosclerosis (MESA). Circulation 2006; 113:30-7.

27. Schroeder S, Achenbach S, Bengel F, et al; Working Group Nuclear Cardiology and Cardiac CT; European Society of Cardiology; European Council of Nuclear Cardiology. Cardiac computed tomography: indications, applications, limitations, and training requirements: report of a Writing Group deployed by the Working Group Nuclear Cardiology and Cardiac CT of the European Society of Cardiology and the European Council of Nuclear Cardiology. Eur Heart J 2008; 29:531-56.

28. Austen WG, Edwards JE, Frye RL, et al. A reporting system on patients evaluated for coronary artery disease. Report of the Ad Hoc Committee for Grading of Coronary Artery Disease, Council on Cardiovascular Surgery, American Heart Association. Circulation 1975; 51(4 Suppl):5-40.

29. Achenbach S. Computed tomography coronary angiography. J Am Coll Cardiol 2006; 48:1919-28

30. World Health Organization. The world health report 2002 - Reducing Risks, Promoting Healthy Life. In: World Health Organization [online]. Available at: http://www.who.int/whr/2002/en/. Accessed June 17, 2015.

31. Víles-Gonzalez JF, Fuster V, Badimon JJ. Atherothrombosis: a widespread disease with unpredictable and life-threatening consequences. Eur Heart J 2004; 25:1197-207.

32. Midiri M, La Grutta L, Grassedonio E, Toia P, Guglielmi G. Non invasive imaging of myocardial infarction with computed tomography and magnetic resonance. Curr Vasc Pharmacol 2015; 13:64-77.

33. Cohen Gl, Aboufakher R, Bess R, et al. Relationship between carotid disease on ultrasound and coronary disease on CT angiography. JACC Cardiovasc Imaging 2013; 6:1160-7.

34. Alluri K, Joshi PH, Henry TS, et al. Scoring of coronary artery calcium scans: history, assumptions, current limitations, and future directions. Atherosclerosis 2015; 239:109-17.

35. Greenland P, Alpert JS, Beller GA, et al; American College of Cardiology Foundation; American Heart Association. 2010 ACCF/AHA guideline for assessment of cardiovascular risk in asymptomatic adults: a report of the American College of Cardiology Foundation/American Heart Association Task Force on Practice Guidelines. J Am Coll Cardiol 2010; 56:e50-103.

36. Abdulla J, Pedersen KS, Budoff M, Kofoed KF. Influence of coronary calcification on the diagnostic accuracy of 64-slice computed tomography coronary angiography: a systematic review and meta-analysis. Int J Cardiovasc Imaging 2012; 28:943-53.

37. Munnur RK, Cameron JD, Ko BS, Meredith IT, Wong DT. Cardiac CT: atherosclerosis to acute coronary syndrome. Cardiovasc Diagn Ther 2014; 4:430-48.

38. Zarins CK, Taylor CA, Min JK. Computed fractional flow reserve (FFTCT) derived from coronary CT angiography. J Cardiovasc Transl Res 2013; 6:708-14.

39. Toth GG, Toth B, Johnson NP et al. Revascularization decisions in patients with stable angina and intermediate lesions: results of the international survey on interventional strategy. Circ Cardiovasc Interv 2014; 7: 751-9.

40. Kim WY, Stuber M, Börnert $P$, et al. Three-dimensional black-blood cardiac magnetic resonance coronary vessel wall imaging detects positive arterial remodeling in patients with nonsignificant coronary artery disease. Circulation 2002; 106:296-9.

41. de Groot E, Jukema JW, Montauban van Swijndregt AD, et al. B-mode ultrasound assessment of pravastatin treatment effect on carotid and femoral artery walls and its correlations with coronary arteriographic findings: a report of the Regression Growth Evaluation Statin Study (REGRESS). J Am Coll Cardiol 1998; 31:1561-7.

42. Hartman J, Frishman $\mathrm{WH}$. Inflammation and atherosclerosis: a review of the role of interleukin- 6 in the development of atherosclerosis and the potential for targeted drug therapy. Cardiol Rev 2014; 22:147-51.

43. Su TC, Jeng JS, Chien KL, et al. Hypertension status is the major determinant of carotid atherosclerosis: a community-based study in Taiwan. Stroke 2001; 32:2265-71. 